\section{Running Gels Backwards to Select DNA Molecules Larger than a Minimum Size}

\section{BioTechniques 30:264-266 (February 2001)}

Many procedures require size selection of DNA molecules for subsequent cloning or for other procedures. If the desired DNA fragments are of a known specific size, then one can easily cut the required band from a gel and extract the DNA; there are many effective methods to purify DNA from the small piece of agarose that contains the band.

It is much less convenient if one needs to select molecules spanning a range of sizes, because they run to different positions in the gel, so that one would need to cut a large piece of agarose to obtain the full range of sizes desired. It is most difficult if one needs molecules larger than a given size; to obtain all such molecules, one would need to cut a piece of agarose extending from the movement limit for large DNA molecules down to a position corresponding to the minimum desired size. Methods that are effective in purifying DNA from small pieces of agarose are often cum bersome for large pieces; even worse, extraction from large pieces of agarose can result in significant amounts of im purities in the extracted DNA.

We have used a simple, fast, and effective procedure to select DNA molecules larger than a given size and to gather them into a small piece of agarose for easy extraction. We run a low-melt agarose gel containing the DNA to be size-selected (along with ethidium bromide and size standards). When the gel has run far enough to accurately locate DNA molecules of the minimum desired size, we cut the gel at that boundary and discard the portion of the gel with molecules that are too small. We replace the running buffer and then run the remaining part of the gel backwards at the same voltage as in the initial run.

At the time the gel is cut, the desired DNA molecules lie in an extended, although possibly very faint, smear extending from the cut toward the well. The position of each molecule in the smear depends on the molecule's mo- bility, which is determined by its size. If the electric current is reversed, so the voltage drop per centimeter of gel is the same as before (but now points in the opposite direction), then the DNA molecules will move back toward the well. Each molecule will move backwards with the same velocity that had previously carried it forward, to its position within the smear when the gel was cut. In effect, the molecules reverse their paths. All of these reversed paths converge at the well, so the smear of DNA gradually compresses into a narrow band as the DNA approaches the well. We monitor the progress of the convergence with a hand-held UV light (models FB-UVM-80 or FB-UVLS-80; Fisher Scientific, Pittsburgh, PA, USA). Shortly before DNA begins to move across the well, we cut the small piece of agarose just in front of the well that contains the converged DNA molecules. We extract the DNA using standard methods; for DNA greater than 2 $\mathrm{kb}$, we find efficient recovery using agarase digestion (Roche Molecular Biochemicals, Basel, Switzerland).

The motion of DNA through a gel includes a random, diffusional component that makes a DNA band gradually thicken as it migrates. Running the DNA backwards does not undo this diffusional spread, so the thickness of the converged band, and the zone within the converged band that is occupied by DNA molecules of each size, reflects the total duration of the run and the distance that the DNA molecules have moved through the gel. As a result, the converged band is at least as thick as an ordinary band of DNA (containing same-sized molecules) that has migrated a comparable total distance through the gel. To minimize this thickness, one may prefer to cut the gel and reverse the migration direction after a shorter rather than longer run. In addition to saving time, this allows the converged band to be excised within a smaller piece of agarose. Without diffusion, optimal convergence would occur precisely at the well, but diffusion limits the minimum thickness of the converged band so all of the DNA does not enter the well at the same time. As a result, the DNA is easiest to excise just before the band enters the well.

In practice, we use the same total voltage when running the gel forward and in reverse. The electrical resistance of the gel is higher than that of the liquid buffer, so removing a portion of the gel lowers the total resistance of the circuit and increases the voltage drop per centimeter of the remaining gel. This somewhat increases the velocity with

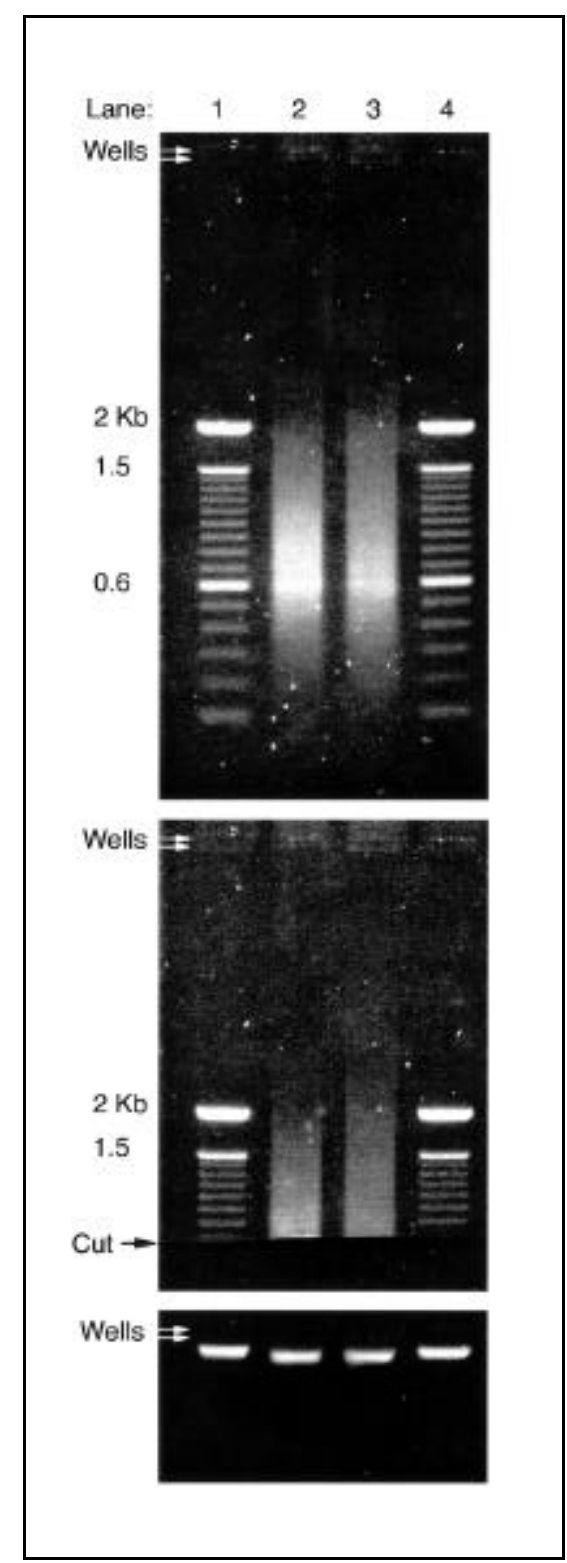

Figure 1. Running a gel backwards to size-select DNA. Lanes 2 and 3 are 5' RACE products. Lanes 1 and 4 are size standards. White arrows show the position of the wells. The top image shows the DNA fully run out (composite of two photos). The middle image shows the gel cut to remove DNA fragments smaller than $700 \mathrm{bp}$. The lower image shows the gel after running it backwards, with DNA converged into narrow bands near the wells. 
which the DNA runs backwards, but the increase is not linear, which should result in an increase in the thickness of the converged band. In our experience, this is not significant compared to the increased thickness due to diffusion, so we do not bother to adjust the voltage to maintain precisely the same voltage drop per centimeter of gel. If the gel is run backwards at a significantly different voltage (e.g., three times as high), then the DNA does not converge into a narrow band. Even with the same total voltage, the time required for the DNA to converge in the backwards run is noticeably shorter than the time needed to run it forward because of the slightly shorter distance run in reverse and because the voltage drop per centimeter of gel is slightly higher. The DNA converges effectively even if as much as two-thirds of the gel is removed, using the same total voltage in reverse.

The rate of DNA migration is strongly affected by the amount of ethidium bromide intercalated within the DNA molecules (1). Ethidium intercalation partially neutralizes the negative charge of the DNA molecule and also increases its length. If ethidium is not present in the gel, the leading DNA molecules gradually lose ethidium and accelerate their motion through the gel. We put sufficient ethidium bromide in the loading dye to saturate the DNA [which can intercalate one ethidium molecule per 2 bp (1)] and put smaller amounts of ethidium in the gel and in the running buffer (both at $400 \mathrm{ng} / \mathrm{mL}$ ) so the DNA remains saturated during the run.

We have found this method especially useful in $5^{\prime}$ rapid amplification of cDNA ends (RACE) to clone apparent full-length transcripts, in spite of significant degradation of the source RNA and a possible strong stop that limits the size of first-strand cDNA synthesis products. Figure 1 shows three images of the same gel with the same $5^{\prime}$ RACE PCR products (Life Technologies, Rockville, MD, USA) in lanes 2 and 3 and 100-bp size standards in lanes 1 and 4. The top image shows the $5^{\prime}$ RACE DNA fully run out. There is a long smear with a partly obscured band at $0.6 \mathrm{kbp}$. Our early cloning efforts had yielded only short partial transcripts of about $0.6 \mathrm{kbp}$. The middle image shows the gel cut to remove fragments smaller than $0.7 \mathrm{kbp}$. The wells are positioned between the white arrows. The lower image shows the gel after running it backwards, with converged DNA bands located close to the wells. TOPO TA cloning (Invitrogen, Carlsbad, CA, USA) of the purified, converged DNA yielded a variety of clones longer than $0.7 \mathrm{kbp}$, including apparent full-length clones of $1.9 \mathrm{kbp}$.

While we typically select DNA larger than a specific size, the method can also be used to obtain DNA between a maximum and a minimum size. The gel is cut perpendicular to the migration direction at positions corresponding to the maximum and minimum sizes of DNA desired. The portion of the gel with smaller fragments is discarded. The gel fragment containing the desired sizes is displaced sideways and held against the part of the gel containing the wells so that the desired DNA moves backwards into an otherwise empty lane. The DNA converges close to the well, as usual. We have found that gels may differ significantly in DNA mobility, even gels that are nominally the same; therefore, we recommend using a portion of the original gel for the backwards migration.

We find this method to be very sim ple and convenient. In addition to $5^{\prime}$ RACE, we also use it to size-select mechanically sheared DNA for subcloning. For highly efficient cloning (as in library construction), it is helpful to minimize UV exposure of the DNA; this can be achieved by covering the part of the gel containing DNA for cloning while the standards are being observed. The desired band is cut when the standard bands converge near the wells.

\section{REFERENCE}

1.Sambrook, J., E.F. Fritsch, and T. Maniatis. 1989. Molecular Cloning: A Laboratory Manual, 2nd ed. CSH Laboratory Press, Cold Spring Harbor, NY.

We thank three anonymous reviewers for their comments. This was supported by $\mathrm{Na}$ tional Science Foundation grant no. DEB9974374 to M.W.F. Address correspondence to Michael W. Frohlich, Herbarium, University of Michigan, 1205 North University, Ann Arbor, MI 48109-1057, USA. e-mail: mfroh@umich.edu
Received 15 May 2000; accepted 7 November 2000.

\author{
Michael W. Frohlich and \\ David S. Parker \\ University of Michigan \\ Ann Arbor, MI, USA
}

\section{Method for the Immuno- logical Detection of Silver- Stained Proteins on Nitro- cellulose Membranes}

\author{
BioTechniques 30:266-272 (February 2001)
}

The combination of western blotting and high-resolution 2-D gel electrophoresis represents a rapid and simple method toward the identification of protein spots in complex mixtures such as lysates from organs, tissues, cells, and body fluids. To obtain a reference 2-D protein spot pattern, it is common to stain all proteins in a gel or on a membrane with silver, taking advantage of the high sensitivity of this dye $(1,4,5)$. Unfortunately, the unequivocal assignment of an immuno-stained spot to the corresponding silver-stained counterpart in 2-D gels is difficult because a method to destain silver-stained proteins on a membrane, and thereby allowing the successive detection by immuno-staining, has not been described. To circumvent this problem, protein mixtures are usually separated in parallel on two 2-D gels that are then blotted. While one of the membranes is subjected to immunological detection, the other membrane is stained with silver (8). How ever, individual handling can lead to regional shrinkage or expansion of the two gels, and as a result, the position of the corresponding protein spots varies to a certain extent (5). This impedes the assignment of an immuno-stained spot to the corresponding silver-stained counterpart. Another method of solving this problem is to visualize the complex protein spot pattern on a 2-D blot with dyes, such 